\title{
Influence of CoQ10 on Autonomic Nervous Activity and Energy Metabolism during Exercise in Healthy Subjects
}

\author{
Aisong ZHENG and Toshio MORITANI* \\ Laboratory of Applied Physiology, Graduate School of Human and Environmental Studies, Kyoto University, \\ Kyoto 606-8501, Japan
}

(Received November 26, 2007)

\begin{abstract}
Summary Background: CoQ10 has come to be widely used as a dietary supplement, and daily intake of it has increased in recent years. CoQ10 is produced in all living organisms and is an essential coenzyme for energy synthesis in the mitochondria and an important scavenger of reactive oxygen species. Objective: This is a randomized, double-blind, placebocontrolled experiment to examine the acute effects of a single dose of CoQ10 on the autonomic nervous system (ANS) by using power spectral analysis of HRV and energy metabolism at rest and during low intensity exercise in healthy subjects. Eleven nonsmoking healthy male students (age: $26 \pm 1 \mathrm{y}$ ) volunteered to participate in this experiment. CM5 lead ECG and gas exchange parameters were recorded $5 \mathrm{~min}$ before, and $30 \mathrm{~min}$ and $60 \mathrm{~min}$ after the oral administration of $\mathrm{CoQ} 10$ or a placebo. Following this, the subjects exercised using a stationary cycle ergometer for $10 \mathrm{~min}$ at $60 \mathrm{rpm}$ with an intensity of $30 \%$ of heart rate reserve. During the exercise, the ECG and gas exchange parameters were recorded continuously. Results: There were no significant differences in heart rate between the CoQ10 and placebo trials at rest or during exercise. With regard to the integrated values of the spectrum, there were no significant differences in the HF power representing parasympathetic activity or LF power representing both sympathetic and parasympathetic nervous activities between the trials at any timepoint. However, during the exercise, HF power and LF power in the CoQ10 trial showed a tendency to increase compared with the placebo trial $(p<0.1)$. Total power representing the over-all ANS activity was significantly increased in the CoQ10 trial during exercise, which implied that autonomic nervous activity was augmented by CoQ10 $(p<0.05)$. CoQ10 also induced enhanced lipid oxidation as shown by the significantly lower respiratory gas exchange ratio $(\mathrm{R})$ and increased fat oxidation during exercise. The results shed some light upon the relationship between the autonomic nervous activity and energy metabolism. Conclusion: These results suggested that CoQ10 may increase fat oxidation with augmented autonomic nervous activity during low intensity exercise.
\end{abstract}

Key Words CoQ10, ANS, energy metabolism, power spectral analysis of HRV

Coenzyme $\mathrm{Q} 10(\mathrm{CoQ} 10)$ is a naturally occurring fatsoluble ubiquinone-10 with vitamin-like properties (1, 2). CoQ10 acts in the body as an antioxidant and may have membrane-stabilizing properties (1). In addition, CoQ10 is an important factor for cellular mitochondrial respiration. CoQ10 acts in part as a redox link between flavoproteins and the cytochromes, which are needed for oxidative phosphorylation and the synthesis of adenosine triphosphate (ATP) (1, 3). In other words, CoQ10 is essential for energy production.

CoQ10 is biosynthesized and concentrated in the heart, kidneys, liver, muscle, pancreas, and thyroid gland. The content of CoQ10 in organs decreases with age (4). Although CoQ10 can be obtained from foods such as meat and fish, its content in them is very low (4). Therefore, some nutritionists have considered CoQ10 suitable as a dietary supplement. CoQ10 is now

*To whom correspondence should be addressed.

E-mail: t.moritani@neuro.mbox.media.kyoto-u.ac.jp widely used as a therapeutic substance to treat a variety of disorders such as ischemic heart disease, Parkinson's disease and diabetes mellitus (5-7). Its supplementation has been touted to improve physical and athletic stamina, muscle fatigue and weakness $(8,9)$.

Currently, there is no information investigating the acute efficacy of CoQ10 supplementation on energy metabolism at rest or during exercise in healthy subjects. In addition, no studies have evaluated the acute effect of CoQ10 on the autonomic nervous system. Previous study has verified that energy metabolism was modulated by the autonomic nervous system (ANS) $(10,11)$. The spectral analysis of heart rate variability (HRV) can evaluate the net effect of sympathetic (SNS) and parasympathetic nervous system (PNS) activity $(12,13)$, namely, autonomic nervous system (ANS) activity.

Accordingly, the purpose of this study was to assess the CoQ10 supplementation on ANS by using power spectral analysis of HRV and energy metabolism at rest 
and during exercise in healthy subjects.

\section{MATERIALS AND METHODS}

Subjects and informed consent. Eleven non-smoking healthy male students (age: $26 \pm 1 \mathrm{y}$; height: $173.3 \pm$ $1.7 \mathrm{~cm}$; body mass: $65 \pm 2.3 \mathrm{~kg}$ ) in Kyoto University volunteered to participate in this randomized, doubleblind, placebo-controlled experiment. This study examined the acute effects of CoQ10 and a placebo on autonomic nervous activity and energy metabolism at rest and during exercise. None of the subjects was taking any medication, and each subject was instructed to avoid beverages containing alcohol or caffeine and strenuous physical activity on the day before the measurements. The experiment administrator explained the purpose of the experiment, test protocol and bioactivity of CoQ10 prior to the experiment to all subjects. Then, informed consent to participate in this study was obtained from each subject. The protocol of the study was approved by the ethical committee of Kyoto University Graduate School.

Study design. Exercise intensity was defined by a target heart rate which was continuously monitored by a heart rate monitor (OEC-6102, Nihon Kohden Co., Japan). Target heart rate was calculated as $30 \%$ of the maximal heart rate reserve by the formula (14):

Target $\mathrm{HR}=($ maximal $\mathrm{HR}-$ resting $\mathrm{HR}) \times 0.3+$ resting $\mathrm{HR}$

Maximal $\mathrm{HR}=220-$ age

Target heart rate was derived from the incremental cycle ergometer (CB-X 1000, NAPS, Japan) test, performed prior to the start of the experiment.

On the day preceding the exercise test, the subjects were instructed to refrain from strenuous physical exercise, tobacco and alcohol. They were instructed not to take any food or beverages during the $10 \mathrm{~h}$ prior to the commencement of exercise.

On the day of the experiment, all experiments were performed in the morning from 8:30 to 11:00 a.m. to minimize the circadian influence. The subject was placed in an upright seated condition for at least $20 \mathrm{~min}$ before the beginning of the experiment in a room at the temperature of $25^{\circ} \mathrm{C}$. ECG and gas exchange parameters for each subject were recorded for $5 \mathrm{~min}$ as baseline (Rest1) data. Then the subjects were provided a simple breakfast (energy content $300 \mathrm{kcal}$ or approximately $1,254 \mathrm{~kJ}$ ), consisting of traditional Japanese food, and then provided one capsule of CoQ10 (30 mg, Nitto Pharmaceutical Industries, Ltd.) or a placebo (cornmeal) with a randomized order after the meal to facilitate absorption of the extremely lipophilic CoQ10. After their taking the capsules at $30 \mathrm{~min}$ (Rest2) and at 60 min (Rest3), ECG and gas exchange parameters were recorded for $5 \mathrm{~min}$ repeatedly. Following this, the subjects exercised using a stationary cycle ergometer (CB-X 1000 , NAPS) for $10 \mathrm{~min}$ at $60 \mathrm{rpm}$ with an intensity of $30 \%$ of heart rate reserve.

During rest, the subjects were instructed to breathe at a frequency of 1 breath every $4 \mathrm{~s}(0.25 \mathrm{~Hz})$ in synchrony with the sound of an electric metronome, as mentioned in our study (15), so that respiratory-linked HRV would not overlap with fluctuations in the low-frequency component. During exercise, the subjects were instructed that they could breathe freely as the respiratory rate would easily exceed the $0.25 \mathrm{~Hz}$, so that low frequency HRV frequency components would not be affected by the respiration.

Data acquisition. The ECG was obtained using a CM5 lead. The analogue output of the ECG was connected to an ECG amplifier (Multi-channel Amplifier MEG-6100, Nihon Kohden Co.) and digitized using a 13 bit analogue-to-digital converter (HTB410) at a sampling of $1 \mathrm{kHz}$, using a $0.5-100 \mathrm{~Hz}$ band pass filter at rest and a $1.5-100 \mathrm{~Hz}$ filter during exercise.

Measurements of gas exchange parameters were obtained using the mixing chamber method (Aero monitor AE-300 S, Minato Medical Science, Japan). The analogue signals of fractional concentrations of $\mathrm{O}_{2}$ and $\mathrm{CO}_{2}$ from the gas analysis and those from the flow transducer were continuously digitized using a 13 bit analogue-to-digital converter at a sampling rate of $50 \mathrm{~Hz}$. The $\dot{\mathrm{V}} \mathrm{O}_{2}$, carbon dioxide production, and expired ventilatory volume were calculated every $15 \mathrm{~s}$.

All signals were stored continuously on a computer (DOS/V) for later analysis.

ECG R-R interval power spectral analysis. The heart acts in a discrete fashion with successive heartbeats leading to a series of fluctuating values of R-R intervals. In recent years, the power spectrum analysis of HRV has been proved to be a reliable non-invasive method to use for the quantitative and qualitative assessment of the activities of the cardiac sympathetic and parasympathetic nervous system in human studies $(10,13)$. In general, power spectrum analysis of HRV has shown two major distinct regions of periodicity in R-R intervals: the high frequency, respiration-linked component (HF, greater than $0.15 \mathrm{~Hz}$ ) and the low frequency component (LF, less than $0.15 \mathrm{~Hz}$ ). The HF is associated solely with activity in the parasympathetic nervous system while LF reflects mainly activity in the sympathetic nervous system, and partly activity in the parasympathetic nervous system $(16,17)$.

In the actual analysis, the derived R-R interval time series was aligned in $2 \mathrm{~Hz}$ sequences for power spectrum analysis. The DC component and linear trends were completely eliminated by digital filtering for band pass between $0.03-0.5 \mathrm{~Hz}$ at rest and $0.03-0.8 \mathrm{~Hz}$ during exercise as described elsewhere $(10,13)$. After passing through a Hamming-type data window, power spectrum analysis using a fast Fourier transform was performed on consecutive 256-s time series of the data for R-R intervals obtained during the experiments. We analysed LF (0.03-0.15 Hz), HF (at rest: 0.15-0.4 Hz, during exercise: $0.15-0.8 \mathrm{~Hz}$ ), and total power (Total, at rest: $0.03-0.4 \mathrm{~Hz}$, during exercise: $0.03-0.8 \mathrm{~Hz}$ ) by integrating the spectrum for the appropriate bandwidth. Because integrated values of the basal spectrum differ greatly among individuals, the value of each placebo trial was standardized as $100 \%$, and the other values were compared to this. 


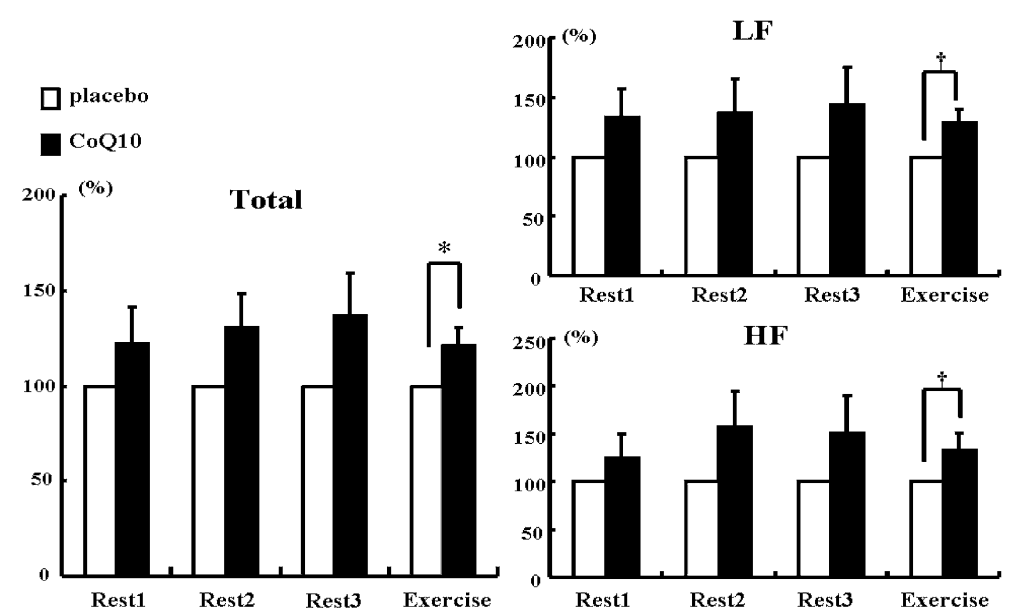

Fig. 1. Changes of activity in the autonomic nervous system following administration of CoQ10 or a placebo at rest and during exercise, as assessed from a power spectrum analysis of the R-R interval. Total: total power of the spectrum, LF: low frequency component of the spectrum, HF: high frequency component of the spectrum. The value of each placebo trial was standardized as $100 \%$. Values represent means (SE), ${ }^{*} p<0.05,{ }^{\dagger} p<0.1$.
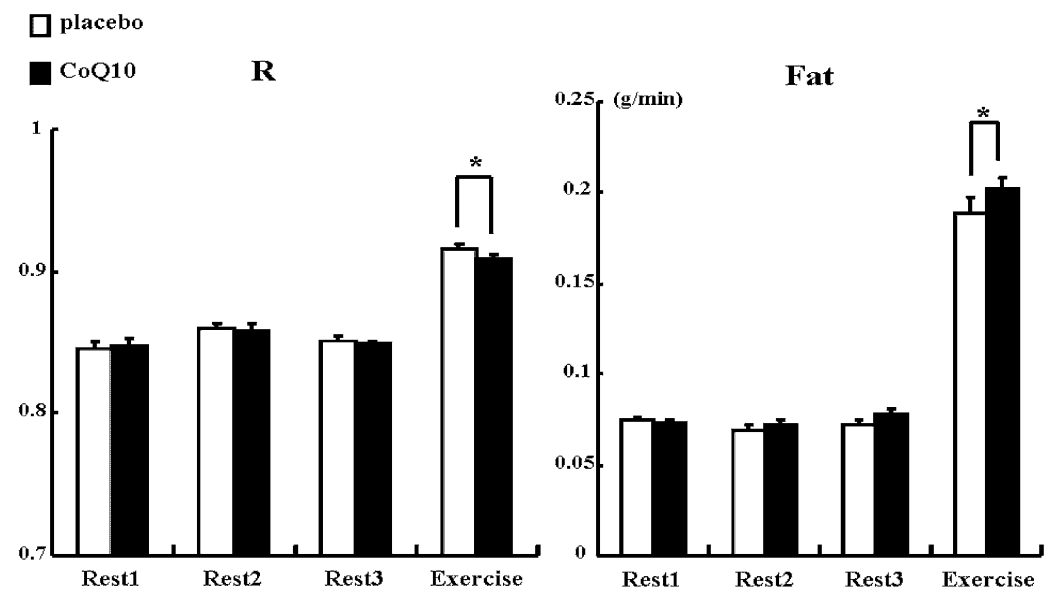

Fig. 2. Changes in energy metabolism after the administration of CoQ10 or a placebo at rest and during exercise. R: respiratory exchange ratio, Fat: fat oxidation $(\mathrm{g} / \mathrm{min})$. Values represent means $(\mathrm{SE}),{ }^{*} p<0.05$.

Statistical analysis. All statistical analyses were performed using a commercial software package (SPSS version 11.5 for Windows, SPSS Inc., Chicago, IL). The effects of time, treatment and time $\times$ treatment were evaluated using 2-way ANOVA for repeated measurements; for comparisons between the trials at certain times, we used Student's paired $t$-test. $p$ values of less than 0.05 were considered to be statistically significant. Data are expressed as mean \pm SE.

\section{RESULTS}

Effect of $\mathrm{CoQ} 10$ on the power spectrum of the R-R intervals

There were no significant differences in heart rate between the CoQ10 and placebo trials at rest or during exercise [Rest1: CoQ10 63 (1.8), placebo 65 (2.2); Rest2: CoQ10 65 (1.2), placebo 66 (2.6); Rest3: CoQ10 65 (1.7), placebo 66 (2.6); exercise: CoQ10 106 (1.3), placebo 108 (2.0) beats/min, means (SE)]. With regard to the integrated values of the spectrum, there were no significant differences in the HF power or LF power between the trials at any timepoint. However, during the exercise, HF power and LF power in the CoQ10 trial showed a tendency to increase compared with the placebo trial [HF 133.5 (18.3)\%, LF 127.5 (13.0)\%, means (SE), $p<0.1]$. Total power showed no significant difference between the trials at rest, but during exercise, total power was significantly increased in the CoQ10 trial [Total $121.5(8.9) \%$, means (SE), $p<0.05$ ] as shown in Fig. 1 .

Metabolic response to $\mathrm{CoQ} 10$

Figure 2 shows the changes in energy metabolism between the CoQ10 and placebo trials at rest and during exercise. $\dot{\mathrm{V}} \mathrm{O}_{2}(\mathrm{~L} / \mathrm{min})$ showed no significant difference between the trials at rest and during exercise. The respiratory gas exchange ratio $(\mathrm{R})$ showed no significant difference between the trials at rest, but during exercise, $\mathrm{R}$ values in the CoQ10 trial were significantly lower than those in the placebo trial [CoQ10 0.91 (0.006), placebo 0.92 (0.002), means (SE), $p<0.05]$. Fat oxidation increased significantly during exercise in the CoQ10 trial compared with the placebo trial [CoQ10 0.20 (0.006), placebo 0.19 (0.008) g/min, 
means (SE), $p<0.05]$.

\section{DISCUSSION}

To the best of our knowledge, this is the first study to examine the effects of CoQ10 supplementation on the autonomic nervous system and energy metabolism during low intensity exercise in healthy subjects.

Since CoQ10 plays a key role in aerobic energy production, elevated serum CoQ10 levels may have a favorable effect on exercising muscles.

It has been reported that CoQ10 increased 2,3diphosphoglycerate levels in erythrocytes (18). Because 2,3-diphosphoglycerate shifts the $\mathrm{Hb}-\mathrm{O}_{2}$ dissociation curve to the right, $\mathrm{O}_{2}$ delivery to the muscles increases at a given $\mathrm{PaO}_{2}$ (19). Therefore, ATP synthesis and lactate production may improve as a result of this increased muscular oxygenation. This could occur not only in skeletal muscle but also in cardiac and respiratory muscles. We speculate that this is the most important mechanism of action of CoQ10.

The autonomic nervous system plays an important role in energy metabolism. A change or reduction in activity of the sympathetic nervous system per se has been widely believed to contribute to the pathogenesis of obesity (20). Shihara et al. (21) have demonstrated that subjects having Trp64 polymorphism of the $\beta-3$ adrenergic receptor mutation (decreased receptor affinity for adrenaline and noradrenaline), responsible for the control of lipolysis through ANS activity, manifested a significantly lower LF power than normal subjects.

Our data showed lower $\mathrm{R}$ values and higher fat oxidation during exercise in the CoQ10 trial compared to those in the placebo trial. Furthermore, our HRV power spectrum data together with the gas exchange data strongly suggest that the CoQ10-induced stimulation of lipid metabolism might be mediated by enhanced overall autonomic nervous activity.

Although there was no significant difference in $\dot{\mathrm{V}} \mathrm{O}_{2}$ values, we found a higher autonomic nervous activity with lower $\mathrm{R}$ values and a higher fat oxidation during exercise after the administration of CoQ10. These changes may have been due to the improved $\mathrm{O}_{2}$ utilization efficiency in peripheral tissue which resulted in enhanced fat metabolism.

Previous studies investigating the effects of CoQ10 supplementation during moderate to high-intensity exercise have been reported. Braun et al. (22) measured the exercise-induced change in $\mathrm{VO}_{2}$ max and lipid peroxidation before and after a one-month CoQ10 supplementation (100 mg daily) in twelve male bicycle racers. As compared with the placebo group, no significant change took place in maximal oxygen consumption, submaximal oxygen consumption, respiratory exchange ratio, heart rate, or total work after CoQ10 supplementation. Roberts (23) also reported no change in maximal oxygen consumption, stroke volume, or cardiac output in sedentary subjects after 4 wk of $100 \mathrm{mg} /$ d CoQ10 supplementation, despite a twofold increase in serum CoQ10 concentration. These results suggested that the choice of exercise protocol-using moderate to high-intensity exercise - may not have been as sensitive to the possible benefits of CoQ10 supplementation as would low-to-moderate-intensity exercise. The present study might confirm that the action of CoQ10 would be sensitive to low intensity exercise during which enhanced fat utilization would take place.

In conclusion, the major finding showed that CoQ10 may be increasing the fat oxidation with augmented autonomic nervous activity during low intensity exercise. The mechanism for this effect is not clear, but CoQ10 administration may useful for the treatment of individuals with hyper-lipidemia or obesity by improving lipolysis. However, because the results of this present study were derived from a small number of subjects, the interpretation of the results should be retested in further larger doses and longer supplementation times with a larger scale study to confirm the present findings.

\section{Acknowledgments}

We would like to thank Nitto Pharmaceutical Industries, Ltd., for providing the supplement. We also thank all the subjects for their participation and cooperation during the experiment.

\section{REFERENCE}

1) Greenberg S, Frishman WH. 1990. Co-enzyme Q10: a new drug for cardiovascular disease. J Clin Pharmacol 30: $596-608$.

2) Mortensen SA, Vadhanavikit S, Muratsu K, Folkers K. 1990. Coenzyme Q10: clinical benefits with biochemical correlates suggesting a scientific breakthrough in the management of chronic heart failure. Int J Tissue React 12: 155-162.

3) Rauchova H, Drahota Z, Lenaz G. 1995. Function of coenzyme $Q$ in the cell: some biochemical and physiological properties. Physiol Res 44: 209-216.

4) Kalen A, Appelkvist EL, Dallner G. 1989. Age-related changes in the lipid compositions of rat and human tissues. Lipids 24: 579-584.

5) Langsjoen H, Langsjoen P, Langsjoen P, Willis R, Folkers K. 1994. Usefulness of coenzyme Q10 in clinical cardiology: a long-term study. Mol Aspects Med 15 (Suppl): S165-S175.

6) Shults CW, Oakes D, Kieburtz K, Beal MF, Haas R, Plumb S, Juncos JL, Nutt J, Shoulson I, Carter J, Kompoliti K, Perlmutter JS, Reich S, Stern M, Watts RL, Kurlan R, Molho E, Harrison M, Lew M; Parkinson Study Group. 2002. Effects of coenzyme Q10 in early Parkinson disease: evidence of slowing of the functional decline. Arch Neurol 59: 1541-1550.

7) Chew GT, Watts GF. 2004. Coenzyme Q10 and diabetic endotheliopathy: oxidative stress and the 'recoupling hypothesis.' QJM 97: 537-548.

8) Kamikawa T, Kobayashi A, Yamashita T, Hayashi H, Yamazaki N. 1985. Effects of coenzyme Q10 on exercise tolerance in chronic stable angina pectoris. Am J Cardiol 56: $247-251$

9) Ogasahara S, Nishikawa Y, Yorifuji S, Soga F, Nakamura Y, Takahashi M, Hashimoto S, Kono N, Tarui S. 1986. Treatment of Kearns-Sayre syndrome with coenzyme Q10. Neurology 36: 45-53.

10) Matsumoto T, Miyawaki T, Ue H, Kanda T, Zenji C, Mori- 
tani T. 1999. Autonomic responsiveness to acute cold exposure in obese and non-obese young women. Int J Obes Relat Metab Disord 23: 793-800.

11) Matsumoto T, Miyawaki $C$, Ue H, Yuasa T, Miyatsuji A, Moritani T. 2000. Effects of capsaicin-containing yellow curry sauce on sympathetic nervous system activity and diet-induced thermogenesis in lean and obese young women. J Nutr Sci Vitaminol 46: 309-315.

12) Akselrod S, Gordon D, Ubel FA, Shannon DC, Berger AC, Cohen RJ. 1981. Power spectrum analysis of heart rate fluctuation: a quantitative probe of beat-to-beat cardiovascular control. Science 213: 220-222.

13) Moritani T, Hayashi T, Shinohara M, Mimasa F, Shibata M. 1993. Comparison of sympatho-vagal function among diabetic patients, normal controls and endurance athletes by heart rate spectral analysis. J Sports Med Sci 7: 31-39.

14) Karvonen MJ, Kentala E, Mustala O. 1957. The effects of training on heart rate; a longitudinal study. Ann Med Exp Biol Fenn 35: 307-315.

15) Hibino G, Moritani T, Kawada T, Fushiki T. 1997. Caffeine enhances modulation of parasympathetic nerve activity in humans: quantification using power spectral analysis. J Nutr 127: 1422-1427.

16) Pomeranz B, Macaulay RJ, Caudill MA, Kutz I, Adam D, Gordon D, Kilborn KM, Barger AC, Shannon DC, Cohen RJ. 1985. Assessment of autonomic function in humans by heart rate spectral analysis. Am J Physiol 248: H151-H153.
17) Pagani M, Lombardi F, Guzzetti S, Rimoldi O, Furlan R, Pizzinelli P, Sandrone G, Malfatto G, Dell'Orto S, Piccaluga E. 1986. Power spectral analysis of heart rate and arterial pressure variabilities as a marker of sympatho-vagal interaction in man and conscious dog. Circ Res 59: 178-193.

18) Sawa H, Takezawa H. 1981. Influence of cellular metabolism on coenzymeQ-10. In: Coenzyme Q-10 Report (Yamamura Y, Kimura E, eds). Eisai Medical View, Tokyo (in Japanese).

19) Kilmartin JV, Rossi-Bernardi L. 1973. Interaction of hemoglobin with hydrogen ions, carbon dioxide, and organic phosphates. Physiol Rev 53: 836-890.

20) Peterson HR, Rothschild M, Weinberg CR, Fell RD, McLeish KR, Pfeifer MA. 1988. Body fat and the activity of the autonomic nervous system. $N$ Engl J Med 318: 1077-1083.

21) Shihara N, Yasuda K, Moritani T, Ue H, Adachi T, Tanaka H, Tsuda K, Seino Y. 1999. The association between Trp64Arg polymorphism of the beta3-adrenergic receptor and autonomic nervous system activity. $J$ Clin Endocrinol Metab 84: 1623-1627.

22) Braun B, Clarkson PM, Freedson PS, Kohl RL. 1991. Effects of coenzyme Q10 supplementation on exercise performance, $\mathrm{VO}_{2} \max$, and lipid peroxidation in trained cyclists. Int J Sport Nutr 1: 353-365.

23) Roberts J. 1990. The effects of Coenzyme Q10 on exercise performance. Med Sci Sports Exerc 22(2): S87. 